\section{Reparación valvular mitral en insuficiencia mitral degenerativa: reparabilidad, resultados inmediatos $y$ seguimiento hasta 20 años}

GONZALO LATORRE S., JOSEFINA ALMEIDA Z. a, SANTIAGO BESA B., SAMUEL CÓRDOVA A., RICARDO ZALAQUETT S.

\section{Mitral valve repair for degenerative mitral insufficiency: likelihood of success, early and-long term results at 20 years}

Background: The 2014 Guidelines for the Management of Patients with Valvular Heart Disease require to know the probability of success and operative mortality of Mitral Valve Repair (MVR) for Degenerative Mitral Insufficiency (DMI) at a given institution. Aim: To assess the probability of success, operative mortality and long-term results of MVR for DMI. Patients and Methods: The database of the Cardiovascular Surgery Service was reviewed for the period December 1991 to December 2013. Long-term survival information was obtained from death certificate records of the Chilean Identification Service. Results: One hundred forty seven patients with DMI were identified, all operated by one author (RZ). In 28 (19\%) the mitral valve was replaced, including three patients in whom a MVR was intended without success. A successful MVR was performed in 119 patients (81\%). The probability of a successful MVR was 97.5\% (119 of 122). Prolapsed posterior leaflet was present in $81 \%$ and annulus dilatation in $60 \%$ of cases. The most frequent surgical procedures were quadrilateral resection (83\%) and chordal transfer (13\%). A mitral annuloplasty was performed in $92 \%$ of cases. Operative mortality was $0.8 \%$. At the end of a $9.9(0-22.7)$ years follow-up, 87 patients (73\%) were alive and mean survival was 16.9 years. Survival rates at 5, 10, 15 and 20 years were 91\%, 78\%, 71\% and 50\%, respectively. Six patients were re-operated, due to mitral valve dysfunction in three. Mean re-operation free survival was 21.4 years. Echocardiographic follow-up was 75\% completed at an average of 64 months; $84 \%$ of cases had no or only 1+ mitral regurgitation. Conclusions: In our experience, MVR for DMI had an operative mortality below $1 \%$ and a probability of success greater than $95 \%$, with excellent long-term results.

(Rev Med Chile 2015; 143: 1233-1241)

Key words: Cardiac surgical procedures; Mitral valve; Mitral valve annuloplasty; Mitral valve insufficiency.

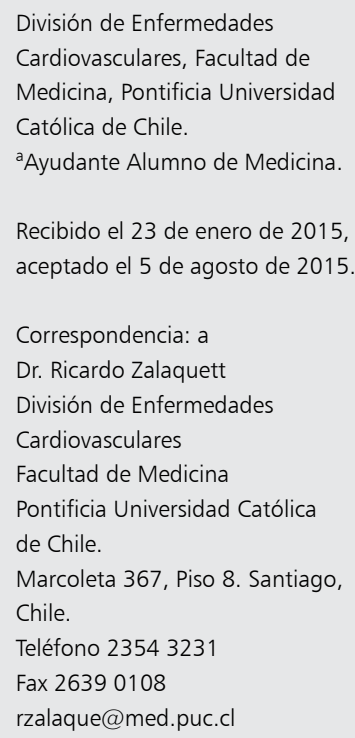<smiles>[Tl]</smiles>
as nuevas guías clínicas conjuntas 2014 de la American Heart Association/American 1 College of Cardiology $y^{1}$ para el manejo de pacientes con enfermedad cardiaca valvular hacen necesario conocer para cada centro cardiovascular, y para cada cirujano en particular, involucrado en el manejo quirúrgico de la insuficiencia mitral degenerativa (IMD), la probabilidad de reparación valvular mitral (RpVM) exitosa y estable en el tiempo, así como la mortalidad operatoria de esta. 
De este modo, las nuevas guías establecen como indicación IIa, con nivel de evidencia B, la RpVM en pacientes asintomáticos con insuficiencia mitral crónica primaria severa y con función ventricular izquierda conservada, si la probabilidad de una reparación mitral exitosa y durable, sin insuficiencia mitral residual, es mayor a 95\%, con una mortalidad operatoria esperada menor a $1 \%$, cuando se efectúa en centros cardiovasculares de excelencia.

La causa más común de insuficiencia mitral crónica primaria, en los países desarrollados o en vías de desarrollo, es el prolapso valvular mitral, cuya etiología más frecuente es la degeneración mixomatosa de la válvula mitral. Esta, a su vez, tiene un amplio espectro de presentación, que va desde una severa degeneración mixomatosa, con compromiso de ambos velos mitrales y cuerdas tendíneas, conocida como enfermedad de Barlow, propia de pacientes jóvenes, a la deficiencia fibroelástiaca, característica de los pacientes mayores, en la que un déficit circunscrito de tejido conectivo origina una ruptura de cuerda tendínea ${ }^{2,3}$.

Nuestra experiencia en cirugía reparadora de la insuficiencia valvular mitral se inicia en 1991, de acuerdo a los principios y técnicas de Carpentier, y con control ecocardiográfico transesofágico intraoperatorio post circulación extracorpórea, en todos los casos, y recientemente comunicamos nuestros resultados globales a 20 años, incluyendo todos los pacientes con insuficiencia mitral primaria y secundaria 4 .

En esta oportunidad comunicamos nuestros resultados a 20 años de la RpVM exclusivamente para la insuficiencia mitral degenerativa, haciendo especial referencia a las exigencias de las nuevas guías clínicas americanas para el manejo de la enfermedad cardiaca valvular, en cuanto a probabilidad de RpVM exitosa y estable en el tiempo y a la mortalidad operatoria esperada de ésta.

\section{Pacientes y Método}

Para la identificación de los pacientes se revisó la base de datos de nuestro Servicio de Cirugía Cardiovascular, para el período comprendido entre diciembre de 1991 y diciembre de 2013. Identificados los pacientes, se revisaron los protocolos operatorios y los informes angiográficos y ecocardiográficos disponibles. Se excluyeron los pacientes con IMD y valvulopatía aórtica o aneurisma de la aorta ascendente asociado. La su- pervivencia alejada se certificó a través del Registro Civil e Identificación de Chile. Todos los pacientes fueron operados por uno de los autores (RZ).

El proyecto fue aprobado por el Comité Ético-Científico de la Facultad de Medicina de la Pontificia Universidad Católica de Chile.

\section{Técnica quirúrgica}

Todos los pacientes tuvieron una RpVM de acuerdo a los principios y técnicas descritos por Alain Carpentier ${ }^{3}$. El resultado de la RpVM fue evaluado intraoperatoriamente post circulación extracorpórea con un ecocardiograma transesofágico efectuado por un cardiólogo ecocardiografista, en todos los casos. Sólo muy excepcionalmente se aceptó una insuficiencia mitral residual mayor a leve $(+)$.

Todos los pacientes fueron operados por esternotomía media con circulación extracorpórea con oxigenador de membrana, con hipotermia sistémica entre $28^{\circ} \mathrm{C} \mathrm{y} 30^{\circ} \mathrm{C}$. La protección miocárdica se efectuó con solución cardioplégica cristaloide (St.Thomas) infundida a $4^{\circ} \mathrm{C}$ por vía anterógrada (raíz aórtica) y retrógrada (seno coronario) ${ }^{5}$.

En todos los casos se utilizó un acceso auricular izquierdo transeptal y superior combinado para abordar la válvula mitral ${ }^{6}$.

\section{Análisis estadístico}

Las variables categóricas se expresan en porcentaje y las variables numéricas en promedio e intervalo de confianza 95\% (IC95\%) o mediana y margen, según su distribución. Las diferencias entre variables numéricas fueron establecidas por medio del test t-Student para variables con distribución normal, demostrada por el método de Kolmogorov-Smirnov. Aquellas variables que no distribuyeron normalmente fueron comparadas mediante el test de rangos de Wilcoxson. Las curvas de supervivencias se construyeron por medio del método de Kaplan-Meier. Un valor alfa de 5\% fue definido a priori para determinar diferencias estadísticas. Este análisis estadístico fue realizado por medio del paquete estadístico SPSS en su versión 17.0 (SPSS Inc., Chicago, USA).

\section{Resultados}

\section{Pacientes}

Se identificaron 147 pacientes con IMD operados por RZ para el período en estudio. En 
Reparación valvular insuficiencia mitral degenerativa - G. Latorre et al

28 casos (19\%) se efectuó un reemplazo mitral, de los cuales en 3 se intentó sin éxito al control ecocardiográfico una RpVM, y en 119 (81\%) una RpVM exitosa. La edad promedio de estos 119 pacientes, en los que se centra el resto de este estudio, fue 60 años (margen: 22 a 82 años). El $65 \%$ (77 pacientes) fueron hombres. La capacidad funcional (NYHA) se consignó en 80 pacientes. De estos, 34\% estaba en CF I-II y $66 \%$ en CF III-IV. La Tabla 1 resume las características preoperatorias de estos pacientes.

\section{Probabilidad RpVM exitosa}

De los 147 pacientes con IMD operados, en 122 se planificó preoperatoriamente una RpVM, de los cuales ésta fue exitosa ecocardiograficamente en 119. Así, la probabilidad de RpVM exitosa fue de 97,5\% (Figura 1).

\section{Degeneración mixomatosa}

La VMD fue tipo deficiencia fibroelástica en 47 casos (40\%) y enfermedad de Barlow en 44 casos (37\%). La Tabla 2 especifica los tipos VMD. La edad promedio de los pacientes con VMD tipo deficiencia fibroelástica fue 64,5 años (IC95\%; $60,7-68,4)$ y de los con enfermedad de Barlow fue $56,7$ años (IC95\%; 56,2-60,8) ( $\mathrm{p}=0,006)$.

\section{Tabla 1. Características basales de los pacientes con reparación valvular mitral exitosa por insuficiencia mitral degenerativa}

\begin{tabular}{|c|c|c|}
\hline & n/media & $\% /$ margen \\
\hline \multicolumn{3}{|l|}{ Sexo } \\
\hline Femenino & 42 & 35 \\
\hline Masculino & 77 & 65 \\
\hline Edad & 60 & $22-82$ \\
\hline \multicolumn{3}{|l|}{ Capacidad funcional* } \\
\hline$|-| \mid$ & 27 & 34 \\
\hline III-IV & 53 & 66 \\
\hline \multicolumn{3}{|l|}{ Grado insuficiencia mitral } \\
\hline Moderada & 3 & 2,5 \\
\hline Severa & 116 & 98 \\
\hline Insuficiencia tricuspídea & 10 & 8,4 \\
\hline Coronariografía & 79 & 66 \\
\hline Enfermedad coronaria & 21 & 27 \\
\hline
\end{tabular}

*Capacidad funcional consignada en 80 pacientes.

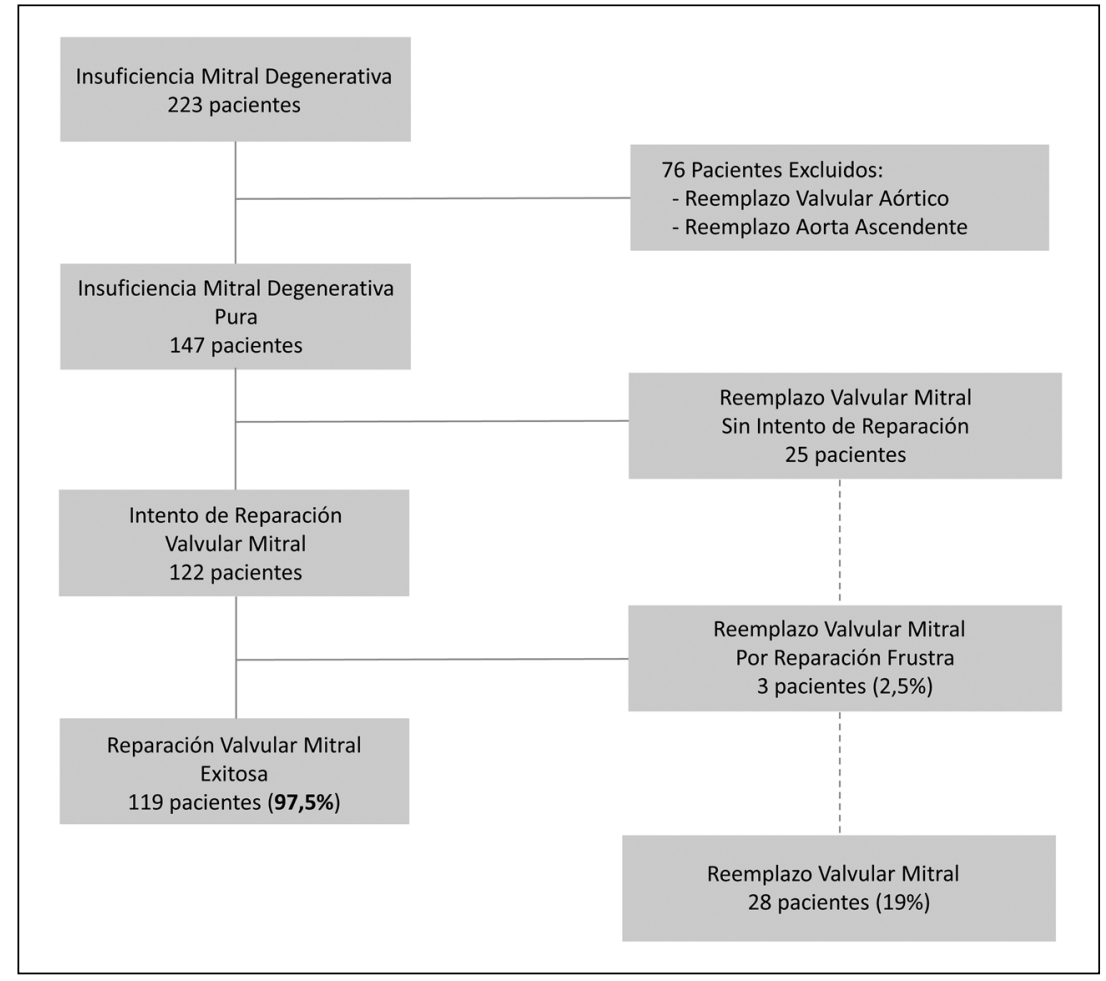

Figura 1. Probabilidad de éxito de la cirugía de reparación valvular mitral por insuficiencia mitral degenerativa. 


\section{Lesión mitral}

La Tabla 3 detalla los tipos de lesión del aparato valvular mitral causante de la insuficiencia mitral. El 81\% de los casos presentaba prolapso del velo posterior, más frecuentemente por rotura de cuerdas tendíneas, y $60 \%$ dilatación del anillo mitral. La mayoría de los pacientes tenían más de una lesión causante de la regurgitación mitral.

\section{Procedimiento quirúrgico}

El procedimiento quirúrgico más frecuente fue la resección cuadrilateral del velo posterior, efectuado en $83 \%$ de los casos, y la transferencia de cuerdas tendíneas, en 13\%. En 109 pacientes (92\%) se efectuó una anuloplastía mitral remodeladora con anillo protésico de Carpentier-Edwards; en 10 casos, como procedimiento exclusivo. La Tabla 4 detalla los procedimientos quirúrgicos efectuados y la Figura 2 los tipos de anillos protésicos utilizados.

\section{Procedimiento quirúrgico asociado}

En 20 pacientes (17\%) se efectuó una revascularización miocárdica asociada, por enfermedad coronaria concomitante, con un promedio de 1,7 puentes/paciente; en 7 se utilizó la arteria mamaria interna izquierda. En 10 pacientes (8\%) se realizó una anuloplastía tricuspídea con anillo de Carpentier-Edwards tricuspídeo y en 45 (38\%) se cerró la orejuela izquierda con una doble sutura corrida de Prolene 3-0 por vía intraauricular de su ostium. En 10 pacientes $(8 \%)$ con fibrilación auricular se efectuó una críocoagulación de venas pulmonares $^{6}$. La Tabla 5 detalla los procedimientos asociados.

\section{Ecocardiografía intraoperatoria}

En $90 \%$ de los casos la evaluación ecocardiográfica intraoperatoria transesofágica post RpVM no demostró insuficiencia mitral residual; en $9 \%$ ésta fue leve y sólo en 2 casos leve a moderada.

\section{Mortalidad operatoria}

Sólo una paciente falleció en el período post operatorio, por una hemorragia cerebral, al inicio de nuestra experiencia. Ella era una paciente hipertensa de difícil manejo y que no se encontraba en tratamiento anticoagulante. Así, la mortalidad operatoria fue $0,8 \%$.
Tabla 2. Tipo de degeneración mixomatosa en pacientes con insuficiencia mitral degenerativa con reparación valvular mitral exitosa

\begin{tabular}{|lcc|}
\hline Tipo degeneración mixomatosa & n & \% \\
\hline Deficiencia fibroelástica & 47 & 40 \\
\hline Enfermedad de Barlow & 44 & 37 \\
\hline Mixta & 24 & 21 \\
Marfanoide & 4 & 3,4 \\
\hline
\end{tabular}

Tabla 3. Hallazgos anatomo-patológicos intraoperatorios en pacientes con insuficiencia mitral degenerativa con reparación valvular mitral exitosa

\begin{tabular}{|lrl|}
\hline Lesiones valvulares & n & \% \\
Prolapso & & \\
Velo posterior & 97 & 81 \\
P1* & 17 & 14 \\
P2* & 66 & 56 \\
P3* & 45 & 38 \\
Velo anterior & 32 & 27 \\
A1* & 9 & 7,6 \\
A2* & 15 & 13 \\
A3* & 15 & 13 \\
Rotura cuerdas tendíneas & 82 & 69 \\
Posterior & 70 & 60 \\
Anterior & 17 & 14 \\
Elongación cuerdas tendíneas & 67 & 56 \\
Posterior & 55 & 46 \\
Anterior & 25 & 21 \\
Dilatación anular & 71 & 60 \\
Perforación & 3 & 2,5 \\
\hline
\end{tabular}

*P1 $=$ segmento anterolateral velo posterior, $\mathrm{P} 2=$ porción media velo posterior, $\mathrm{P} 3=$ porción posteromedial velo posterior, $A 1=$ segmento anterolateral velo anterior, $A 2=$ porción media velo anterior, $\mathrm{A} 3=$ porción posteromedial velo anterior.

Tabla 4. Técnicas quirúrgicas utilizadas en cirugía de reparación valvular mitral en pacientes con insuficiencia mitral degenerativa

\begin{tabular}{|lcc|}
\hline Técnica quirúrgica & n & \% \\
\hline Anuloplastía & 109 & 92 \\
\hline Anuloplastía exclusiva & 10 & 8,4 \\
\hline Resección cuadrilateral del velo posterior & 99 & 83 \\
\hline Transferencia cuerdas tendíneas & 15 & 13 \\
\hline Acortamiento cuerdas tendíneas & 4 & 3,4 \\
\hline Cierre cleft & 6 & 5 \\
\hline
\end{tabular}




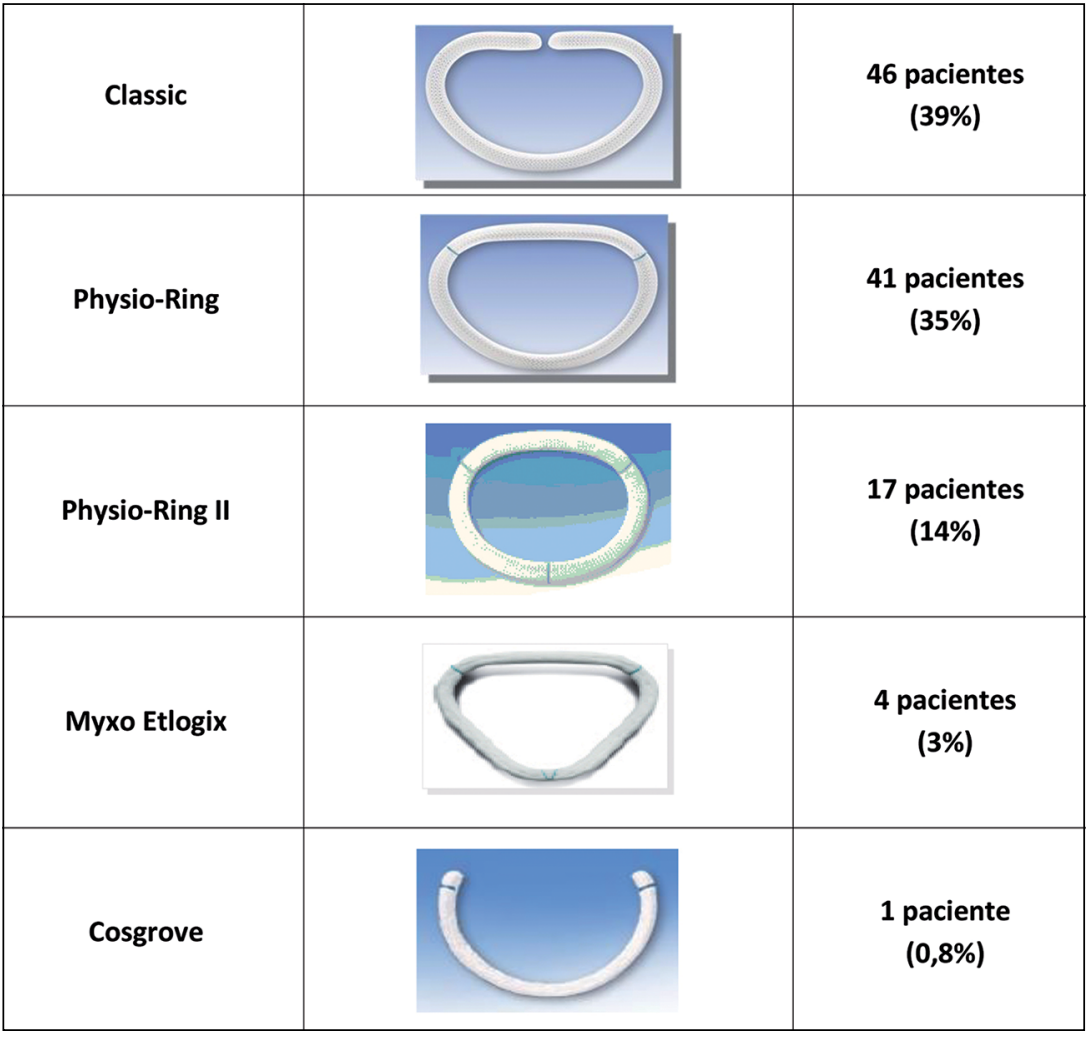

Figura 2. Anillos protésicos mitrales Carpentier-Edwards utilizados en cirugía de reparación valvular mitral por insuficiencia mitral degenerativa (109 pacientes).

\section{Seguimiento alejado}

El seguimiento se completó en $100 \%$ de los casos, con un promedio de 9,9 años ( 0 a 22,7 años). $\mathrm{Al}$ término del seguimiento, 87 pacientes (73\%) estaban vivos. La supervivencia promedio fue 16,9 años (IC95\%; 15,4-18,5). La supervivencia global

\section{Tabla 5. Procedimientos asociados a la cirugía de reparación valvular mitral en pacientes con insuficiencia mitral degenerativa}

\begin{tabular}{|lrc|}
\hline Procedimientos asociados & n & \% \\
\hline Cirugía de revascularización miocárdica & 20 & 17 \\
Arteria mamaria interna izquierda & 7 & 5,9 \\
1 Puente aortocoronario & 10 & 8,4 \\
2 Puentes aortocoronarios & 6 & 5,0 \\
3 o más puentes aortocoronarios & 3 & 2,5 \\
Arteria radial & 1 & 0,8 \\
Cierre orejuela izquierda & 45 & 38 \\
Anuloplastía tricuspídea & 10 & 8,4 \\
Criocoagulación venas pulmonares & 10 & 8,4 \\
\hline
\end{tabular}

a 5, 10, 15 y 20 años fue 91\%, 78\%, 71\% y 50\%, respectivamente (Figura 3 ). De los 32 pacientes fallecidos durante el seguimiento, 16 pacientes (50\%) fallecieron por causa cardiaca y $16(50 \%)$ por causa no cardiaca. Así, la supervivencia alejada libre de muerte cardiaca a 5, 10, 15 y 20 años fue $95 \%, 89 \%, 83 \%$ y $70 \%$, respectivamente (Figura 4 ).

\section{Variables asociadas a mortalidad}

Se realizó un análisis multivariado para identificar las variables asociadas a mortalidad alejada, el que se detalla en la Tabla 6. De acuerdo a este, sólo la edad se asoció a mortalidad. Por el contrario, el cierre de la orejuela izquierda se identificó como un factor protector de mortalidad.

\section{Seguimiento ecocardiográfico}

El seguimiento ecocardiográfico se completó en $75 \%$ de los casos ( 89 pacientes), con un promedio de 64 meses (IC95\%; 51,2-77,6). El 84\% de los casos no tenía insuficiencia mitral o ésta era leve $(1+)$ (Tabla 7$)$. 

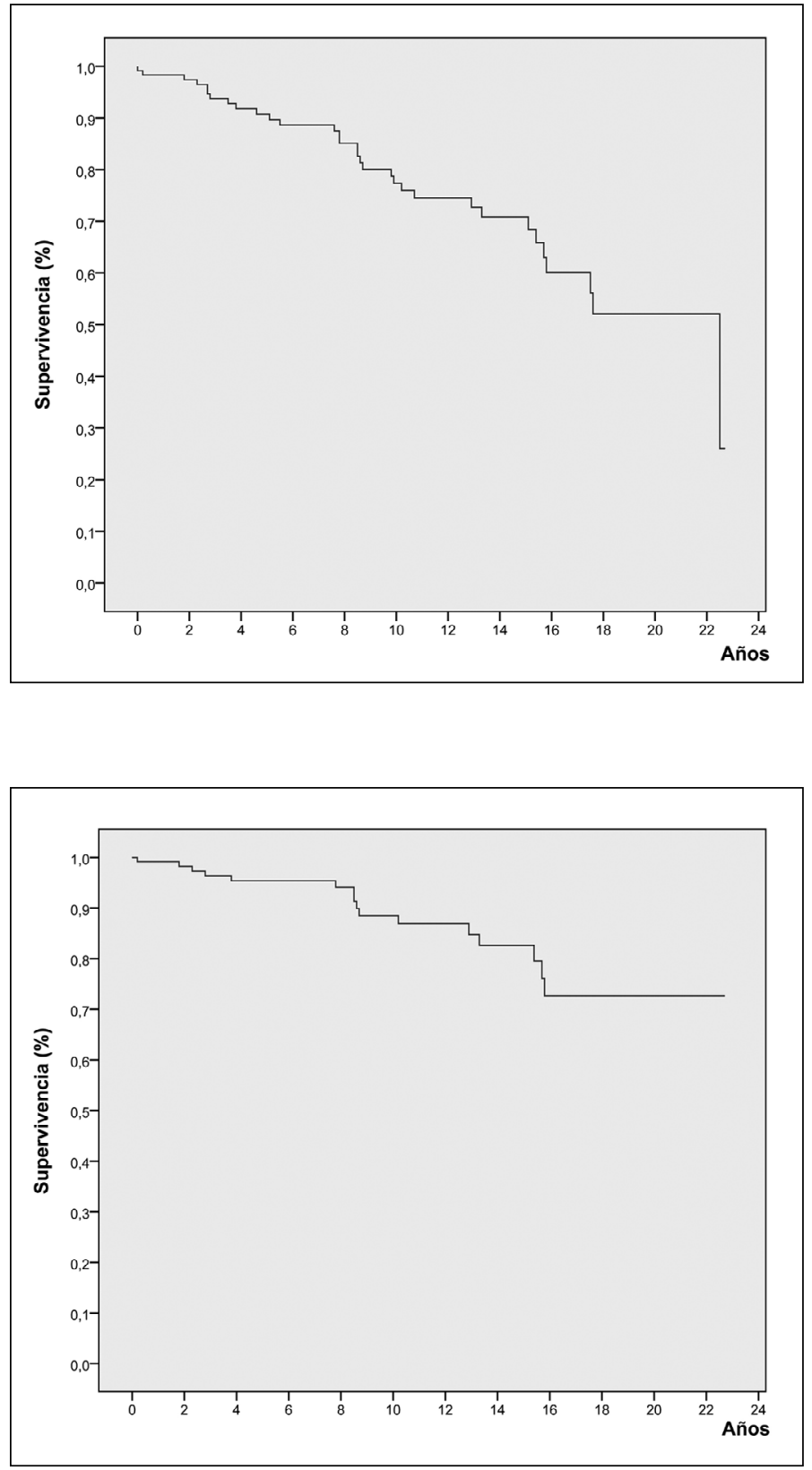

Figura 3. Supervivencia global post cirugía de reparación valvular mitral por insuficiencia mitral degenerativa estimada por método de Kaplan-Meier.

Figura 4. Supervivencia libre de muerte cardiaca post cirugía de reparación valvular mitral por insuficiencia mitral degenerativa estimada por método de Kaplan-Meier.

Tabla 6. Análisis multivariado de principales variables asociadas a mortalidad alejada de cirugía de reparación valvular mitral en pacientes con insuficiencia mitral degenerativa

\begin{tabular}{|lccccc|}
\hline & B & Wald & OR & IC95\% & Valor p \\
\hline Edad * & 0,098 & 16,2 & 1,1 & $1,05-1,16$ & $<0,001$ \\
\hline Cierre de orejuela* & $-2,03$ & 11,5 & 0,13 & $0,04-0,4$ & 0,001 \\
\hline
\end{tabular}

*Ajustados por sexo y comorbilidades. 


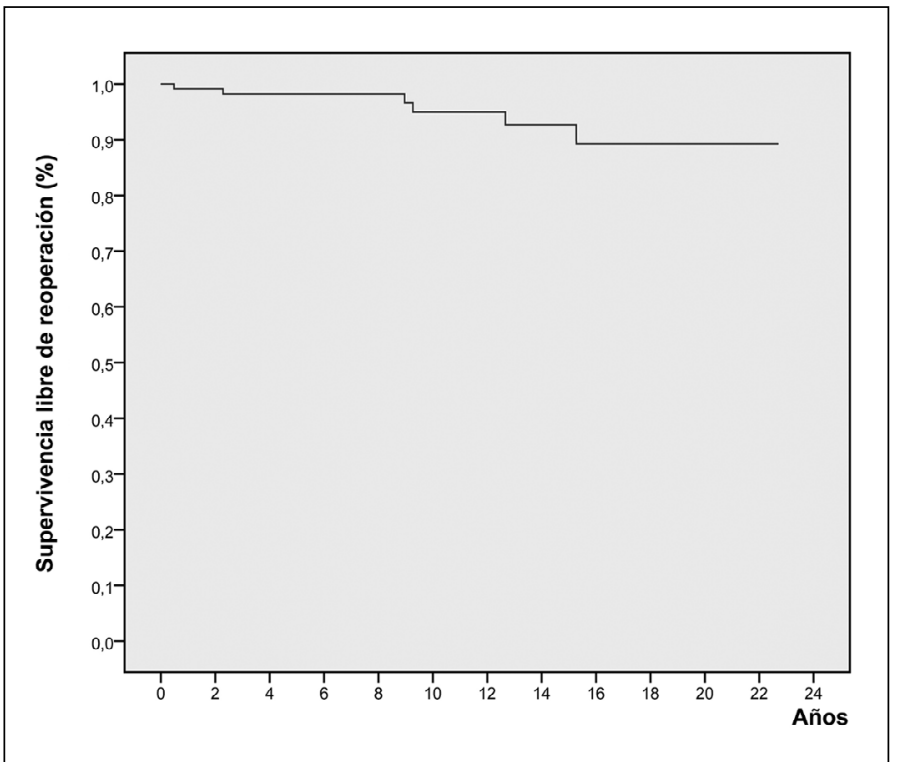

Figura 5. Supervivencia libre de reoperación mitral post cirugía de reparación valvular mitral por insuficiencia mitral degenerativa estimada por método de Kaplan-Meier.
Tabla 7. Control ecocardiográfico alejado de pacientes con cirugía de reparación valvular mitral por insuficiencia mitral degenerativa

\begin{tabular}{|lcc|}
\hline Insuficiencia mitral & n & \% \\
\hline Mínima o ausente & 52 & 58 \\
Leve & 23 & 26 \\
Moderada & 12 & 14 \\
Severa & 2 & 2 \\
\hline
\end{tabular}

\section{Reoperación}

Seis pacientes (5\%) se reoperaron en nuestra institución durante el seguimiento, pero sólo 3 $(2,5 \%)$ de ellos de la válvula mitral (2 por endocarditis y 1 por recidiva de la insuficiencia mitral). En estos 3 casos se efectuó un recambio mitral. De los otros 3 pacientes, 2 se reoperaron por valvulopatía aórtica y 1 por una disección aórtica. Así, el tiempo promedio libre de reoperación mitral fue 21,4 años (IC95\%;20,4-22,4) y la probabilidad de estar vivo libre de reoperación valvular mitral a 5, 10, 15 y 20 años fue $98 \%, 95 \%, 93 \%$, y $88 \%$, respectivamente (Figura 5).

\section{Discusión}

La enfermedad degenerativa de la válvula mitral es en la actualidad la principal causa de insuficiencia mitral en los países desarrollados o en vías de desarrollo ${ }^{1}$. El espectro anatomopatológico de la degeneración mixomatosa es muy amplio y va desde cambios histológicos localizados a la porción media del velo posterior, habitualmente con rotura de una o a lo más 2 cuerdas tendíneas, lo que se conoce como deficiencia fibrolelástica, descrita por Alain Carpentier, a un compromiso degenerativo extenso de todo el aparato valvular mitral, con velos gruesos y voluminosos, cuerdas tendíneas laxas y elongadas, dilatación, e incluso calcificación del anillo mitral, lo que se conoce como enfermedad de Barlow ${ }^{2,3}$. Desde ya, es importante señalar que la diferenciación entre estas 2 formas extremas de degeneración mixomatosa de la válvula mitral tiene importantes implicancias quirúrgicas.

Igualmente, en la actualidad, la RpVM es considerada el tratamiento de elección para los pacientes con IMD sintomática ${ }^{1,7,8}$. Sin embargo, en pacientes asintomáticos la situación es más controvertida. Si bien no existen estudios prospectivos y aleatorios que comparen la RpVM versus la observación clínica, varios estudios observacionales o retrospectivos en pacientes asintomáticos con insuficiencia mitral severa demuestran que la RpVM mejora la supervivencia alejada de estos pacientes ${ }^{9-12}$. Las guías clínicas americanas y europeas, publicadas a mediados 
de la década recién pasada, sugieren que se podría considerar la RpVM en pacientes asintomáticos si la posibilidad de una reparación exitosa es alta y la mortalidad operatoria baja ${ }^{7,8}$. Las nuevas guías clínicas conjuntas 2014 del AHA/ACC para el manejo de la enfermedad valvular cardiacas son ahora muy explícitas a este respecto. Así, estas guías establecen que la RpVM es una indicación quirúrgica razonable en pacientes asintomáticos con IMD crónica severa y función ventricular izquierda conservada (fracción de eyección > $60 \%$ y diámetro de fin de sístole $<40 \mathrm{~mm}$ ), si la probabilidad de una RpVM exitosa y durable, sin insuficiencia mitral residual, es mayor a $95 \%$ y con una mortalidad operatoria esperada menor a $1 \%$, cuando es efectuada en un centro valvular cardiaco de excelencia ${ }^{1}$. De este modo, es necesario para cada centro cardiovascular, y, para cada cirujano en particular, conocer cuáles son sus posibilidades de efectuar una RpVM exitosa y estable y cuál es su mortalidad operatoria, en pacientes asintomáticos con IMD severa con función ventricular izquierda normal. El dar cumplimiento a estas obligaciones fue el principal objetivo de este trabajo.

\section{Características de los pacientes}

La edad de los pacientes fue muy similar a otras series reportadas, en especial a la de David et $\mathrm{al}^{13}$, con claras diferencias entre los pacientes con IMD tipo deficiencia fibroelástica y aquellos con enfermedad de Barlow, siendo los primeros en promedio 8 años más viejos. Igualmente, la mayoría de los pacientes eran hombres, y si bien también la mayoría eran sintomáticos, un tercio de estos eran asintomáticos o poco sintomáticos. Todo lo anterior hace comparable y valedero nuestros resultados.

\section{Lesión mitral y procedimiento quirúrgico}

Como en la mayoría de las series, la lesión más frecuente fue el prolapso del velo posterior, $y$, en la mayoría de estos casos, secundaria a rotura de cuerdas tendíneas. De hecho, en nuestra opinión, es este tipo de lesión, en especial cuando se circunscribe a la porción media del velo posterior (P2), la más favorable para una RpVM exitosa, a través de una simple resección cuadrilateral. Como ya hemos señalado, nuestra experiencia se ha basado en seguir los principios establecido por Carpentier en forma estricta, por lo que hemos evitado las plicaturas o resecciones triangulares así como el uso de cuerdas tendíneas artificiales ${ }^{3}$. De igual modo, sólo en una ocasión no utilizamos un anillo protésico completo de Carpentier-Edwards, puesto que consideramos que en la RpVM por IMD la anuloplastía es un complemento de la reparación que persigue remodelar, no reducir, el anillo mitral nativo, devolviéndole su forma natural, la que se asemeja a un riñón, estabilizando de esta forma la reparación.

\section{Probabilidad de RpVM exitosa}

En esta experiencia de más de 20 años, la mayoría (81\%) de los pacientes con IMD tuvieron una RpVM exitosa, sin insuficiencia mitral residual. Pero, más importante aun, en los pacientes en que se planificó preoperatoriamente un RpVM, ésta fue exitosa en 97,5\% de ellos al control ecocardiográfico intraoperatorio. De este modo, nuestra experiencia satisface las exigencias de las nuevas guías clínicas norteamericanas ${ }^{1}$.

\section{Mortalidad operatoria}

Nuevamente, nuestra experiencia cumple con la exigencia de las guías clínicas señaladas al ser la mortalidad operatoria menor a $1 \%{ }^{1}$. Esto, a pesar de que en la mayoría de nuestros pacientes en los que se consignó la capacidad funcional, ésta era III-IV. Más aun, el único caso de mortalidad se produjo muy al comienzo de nuestra experiencia.

\section{Supervivencia y estabilidad de la reparación}

La RpVM les ofreció a nuestros pacientes una excelente supervivencia alejada, estando 73\% de ellos vivos al término del seguimiento, completado en $100 \%$ de los casos, con una supervivencia promedio de casi 17 años. Igualmente destacable, la supervivencia libre de muerte cardiaca a 10 y 20 años fue $89 \%$ y $70 \%$, respectivamente.

Como en nuestra publicación previa ${ }^{4}$, que comprende todos los pacientes con RpVM, el cierre de la orejuela izquierda apareció como un factor protector de mortalidad alejada, para lo cual no tenemos una explicación clara y sólo podemos elucubrar una menor probabilidad de eventos trombo-embólicos.

Por otra parte, $84 \%$ de los pacientes a un seguimiento ecocardiográfico promedio de 64 meses, no tenía insuficiencia mitral o ésta era leve, si bien en este caso el seguimiento no fue $100 \%$ completo, lo que se compara favorablemente con series grandes internacionales ${ }^{9,10,13}$. 
La probabilidad de estar vivo libre de reoperación mitral fue igualmente muy buena, siendo $95 \%$ y $88 \%$ a 10 y 20 años, respectivamente, si bien esto considera sólo los pacientes reoperados en nuestra institución.

\section{Limitaciones del estudio}

El trabajo presenta las limitaciones propias de los estudios observacionales retrospectivos, las que pueden acentuarse al ser el período de observación muy largo, de más de 20 años. Lo anterior, se hizo manifiesto en el seguimiento ecocardiográfico, el que no se completó en $100 \%$ de los casos, y en la información respecto a reoperación, la que sólo se pudo obtener en pacientes reoperados en nuestra institución.

\section{Conclusión}

En esta experiencia de más de 20 años, $81 \%$ de los pacientes con IMD recibió como tratamiento de esta una RpVM. De los pacientes en que preoperatoriamente se planificó una RpVM, esta fue exitosa en $97,5 \%$ de los casos y la mortalidad operatoria fue $0,8 \%$, con lo que se cumple a cabalidad con las exigencias de las guías clínicas conjuntas AHA/ACC $2014^{1}$.

\section{Referencias}

1. Nishimura RA, Otto CM, Bonow RO, Carabello BA, Erwin JP, Guyton RA, et al. 2014 AHA/ACC guideline for the management of patients with valvular heart disease: Executive summary: A report of the American College of Cardiology/American Herat Association Task Force on Practice Guidelines. Circulation 2014; 129: $1-96$.

2. Adams DH, Anayanwu AC. Seeking a higher standard for degenerative mitral valve repair: begin with etiology. J Thorac Cardiovasc Surg 2008; 136: 551-6.

3. Carpentier A. Cardiac valve surgery-the "French correction”. J Thorac Cardiovasc Surg 1983; 86: 323-37.

4. Zalaquett R, Latorre G, Almeida J, Meneses A, Muñoz
C, Córdova S. [Twenty years of mitral valve repair. An experience in 322 patients]. Rev Med Chile 2014; 142: 1089-98.

5. Zalaquett R, Becker P, Irarrázaval MJ, Moran S, Maturana $G$, Navarro $M$, et al. Cardioplegia retrógrada por canulación transauricular derecha del seno coronario. Rev Chil Cardiol 1993; 12: 68-71.

6. Zalaquett R, Pérez E, Garayar B, Vergara I, Moran S, Becker $\mathrm{P}$, et al. Criocoagulación de venas pulmonares como tratamiento complementario de la fibrilación auricular en cirugía valvular. Rev Med Chile 2007; 135: 871-8.

7. Bonow RO, Carabello BA, Chatterjee K, deLeon AC, Faxon DP, Free MD, et al. ACC/AHA 2006 Guidelines for the management of patients with valvular heart disease: A report of the American College of Cardiology/ American Heart Association Task Force on Practice Guidelines. Circulation 2006; 114: e84-e231.

8. Vahanaian A, Baumgartner H, Bax J, Butchart E, Dion $\mathrm{R}$, Filippatos $\mathrm{G}$, et al. Guidelines of the management of valvular heart disease. The task force on the management of valvular heart disease of the European Society of Cardiology. Eur Heart J 2007; 28: 230-68.

9. Ling LH, Enríquez-Sarano M, Seward JB, Orzulak TA, Schaff HV. Bailey KR, et al. Early surgery in patients with mitral regurgitation due to flail leaflets: a long-term outcome study. Circulation 1997; 96: 1819-25.

10. David TE, Ivanov J, Armstrong S, Rakowski H. Late outcomes of mitral valve repair for floppy valves: Implications for asymptomatic patients. J Thorac Cardiovasc Surg 2003; 125: 1143-52.

11. Gillinov AM, Mihaljevic T, Blackstone EH, George K, Svensson LG, Nowicki ER, et al. Should patients with severe degenerative mitral regurgitation delay surgery until symptoms develop? Ann Thorac Surg 2010; 90: 481-8.

12. Kang DH, Kim JH, Rim JH, Kim MJ, Yung SC, Song JM, et al. Comparison of early surgery versus conventional treatment in asymptomatic severe mitral regurgitation. Circulation 2009; 119: 797-804.

13. David TE, Armstrong S, McCrindle BW, Manlhiot C. Late outcomes of mitral valve repair for mitral regurgitation due to degenerative disease. Circulation 2013; 127: 1485-92. 